\title{
Extraction and Application of Expert Priors to Combine Multiple Segmentations of Human Brain Tissue
}

\author{
Torsten Rohlfing, Daniel B. Russakoff, and Calvin R. Maurer, Jr. \\ Image Guidance Laboratories, Department of Neurosurgery, Stanford University, \\ 300 Pasteur Drive, MC 5327, Stanford, CA 94305-5327, USA \\ \{rohlfing, dbrussak\}@stanford.edu, calvin.maurer@igl.stanford.edu
}

\begin{abstract}
This paper evaluates strategies to combine multiple segmentations of the same image, generated for example by different segmentation methods or by different human experts. Three methods are compared, each estimating and using a different level of prior knowledge about the segmenters. These three methods are: simple label averaging (no priors), a binary expectation maximization (EM) method with independent per-label priors [Warfield et al., MICCAI 2002], and a simultaneous multi-label EM method with across-label priors [Rohlfing et al., IPMI 2003]. The EM methods estimate the accuracies of the individual segmentations with respect to the (unknown) ground truth. These estimates, analogous to expert performance parameters, are then applied as weights in the actual combination step. In the case of the multi-label EM method, typical misclassification behavior, caused for example by neighborhood relationships of different tissues, is also modeled. A validation study using the MNI BrainWeb phantom shows that decision fusion based on the two EM methods consistently outperforms label averaging. Of the two EM methods, the multi-label technique produced more accurate combined segmentations than the binary method. We conclude that the EM methods are useful to produce more accurate segmentations from several different segmentations of the same image.
\end{abstract}

\section{Introduction}

Image segmentation can be looked at as a classification problem: a segmentation method receives a voxel coordinate as its input, and based on the image information at that location returns a classification (labeling) of the voxel. It is well known in the pattern-recognition community that a combination of classifiers can provide better results than one classifier alone, especially if they make different errors and thereby complement each other 112. In recent work on atlas-based segmentation, our group has shown that the accuracy of atlas-based segmentation by means of non-rigid registration [4] can be substantially improved by combining segmentations obtained using multiple atlases [3].

These findings immediately motivate the search for an optimal method to integrate multiple segmentations of the same image into one by combining the 


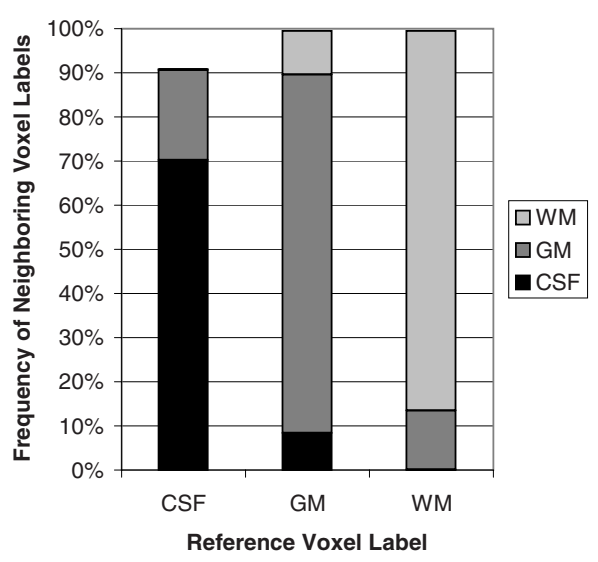

(a) A priori distribution

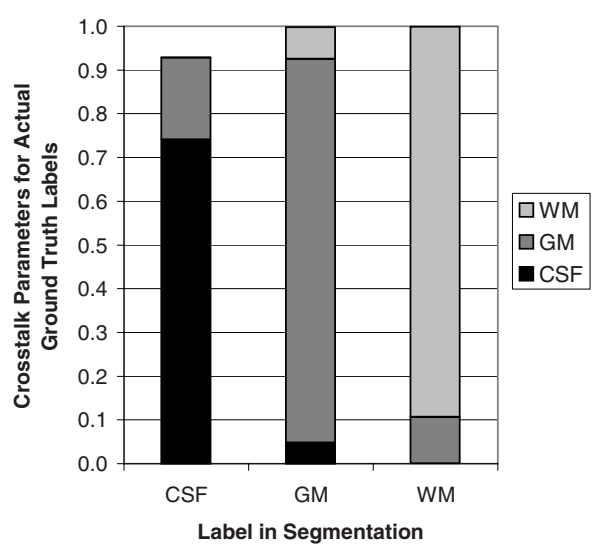

(b) Expert parameters determined by multi-label EM algorithm

Fig. 1. Comparison of tissue neighborhood statistics in the MNI BrainWeb Phantom for CSF, GM, and WM. (a) A priori distribution computed by counting voxel labels in 3 -D neighborhoods (26 neighbors). (b) Crosstalk weights $\lambda_{i, j}$ for one classifier as determined by the multi-label EM algorithm.

decisions of multiple classifiers in a way that achieves the best classification accuracy. A trivial way to approach the problem in image segmentation is label averaging (vote fusion), whereby for a voxel each of the individual segmentations "votes" for one label, and the label with the highest number of votes is assigned to that voxel in the final segmentation.

For binary (i.e., foreground vs. background) segmentation, Warfield et al. [5] have recently introduced an expectation maximization (EM) algorithm for simultaneously estimating the performance parameters of several experts, as well as the unknown ground truth. Their algorithm can also be understood (and used) as a method to combine multiple segmentations into one (the ground truth estimate). In doing so, the expert parameters estimated by this method serve as natural weights in the soft combination of the classifiers that are the experts [6].

Two recently proposed extensions [7] to the Warfield algorithm are capable of generating a combined segmentation from several multi-label segmentations and estimating the corresponding expert parameters. One method applies the Warfield algorithm to each label separately and subsequently resolves ambiguities to generate a combined segmentation. The second method is a true multilabel extension that treats all labels simultaneously. Both methods were assessed in an earlier validation study using biomedical atlases [7, which distinguish 22 anatomical structures. It was found that repeated application of the binary algorithm produced segmentation accuracies superior both to the simultaneous multi-label algorithm and simple label averaging. 

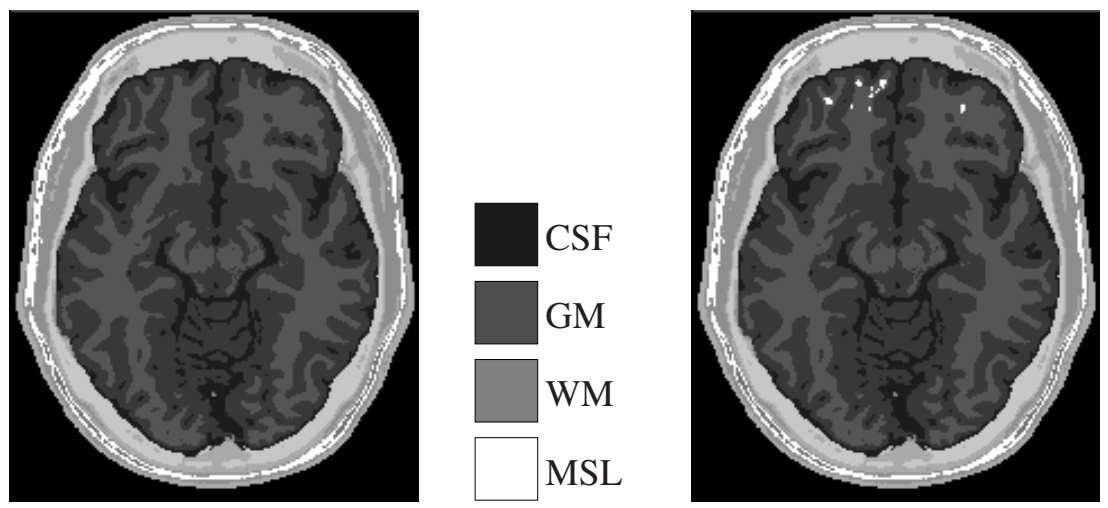

Fig. 2. Representative axial slices form the MNI BrainWeb phantom atlas. Left: normal, right: with simulated multiple sclerosis lesions. The color scale shows the gray values that represent cerebrospinal fluid (CSF), gray matter (GM), white matter (WM), and multiple sclerosis lesions (MSL; right image only).

The present paper is based on the hypothesis that the situation is different when, for example, one is interested in labeling the different tissue types within the human brain. Due to the structure of the brain, certain neighborhood relationships between labels occur substantially more frequently than others. For example, among the three tissue types cerebrospinal fluid (CSF), white matter (WM), and gray matter (GM), the combinations CSF-GM and GM-WM occur substantially more often than CSF-WM (see Fig. 1(a)). Therefore, misclassifications of a voxel are typically not independent of the ground truth for that voxel. Instead, one can assume the existence of expert priors that quantify the inter-dependence of the ground truth segmentation and the distribution of misclassifications. We demonstrate in this paper how such expert priors can be automatically deduced by our simultaneous multi-label EM algorithm in order to combine multiple brain tissue segmentations into one that most accurately reflects the correct labeling.

\section{Multi-classifier Segmentation}

The general idea of multi-classifier segmentation is to combine the decisions $e_{k}(x)$ of the $K$ classifiers (or experts) 1 through $K$ for a voxel $x$ into a final segmentation $E(x)$. For the present paper, three different multi-label atlas combination methods were tested: label averaging ("AVG"), independent binary EM ("EMB"), and simultaneous multi-label EM ("EMM").

\subsection{Label Averaging (AVG)}

For each voxel $x$, the labels $e_{k}(x)$ assigned by all segmentations $k$ are counted. The most frequently assigned label is assigned to that voxel in the final segmentation. In other words, the final segmentation $E(x)$ for $x$ is 


$$
E(x)=\arg \max _{j} \sum_{k: e_{k}(x)=j} 1 .
$$

This procedure, which uses no estimate of the expert performance, is commonly referred to as decision combination by vote fusion [2].

\subsection{Binary Expectation Maximization (EMB)}

The EM algorithm described by Warfield et al. [5] is applied to each label separately. The method estimates the common expert parameters $p$ (sensitivity) and $q$ (specificity), i.e., the fraction of true positives and true negatives among the classified voxels. The parameters $p$ and $q$ are estimated separately for each expert $k$ and each class $C_{i}$ (label in the ground-truth segmentation) as the following conditional probabilities:

$$
p_{i}^{(k)}=P\left(e_{k}(x)=i \mid x \in C_{i}\right) \text { and } q_{i}^{(k)}=P\left(e_{k}(x) \neq i \mid x \notin C_{i}\right) .
$$

From these expert parameters, an EM algorithm can be derived as described by Warfield et al. 5. Since in this method there is no interaction between the computations for different labels, the combination of ground truth estimates for all labels may be ambiguous. However, from the computed expert parameters for each label, a contradiction-free final segmentation $E$ can be computed as

$$
E(x)=\arg \max _{i} P\left(x \in C_{i} \mid e_{1}(x), \ldots, e_{K}(x)\right) .
$$

Here, the probability $P\left(x \in C_{i} \mid \mathbf{e}\right)$ follows from the experts' decisions and their performance parameters using Bayes' rule. For details on the application of this algorithm to classifier fusion, see Ref. [7].

\subsection{Multi-label Expectation Maximization (EMM)}

In a generalization of the Warfield algorithm to multi-label segmentations [7], the expert parameters $p$ and $q$ are replaced by a matrix of label cross-segmentation coefficients $\lambda_{i, j}^{(k)}$. These describe the conditional probabilities that for a voxel $x$ in class $C_{i}$ the expert $k$ assigns label $j=e_{k}(x)$, that is

$$
\lambda_{i, j}^{(k)}=P\left(e_{k}(x)=j \mid x \in C_{i}\right) .
$$

This formulation includes the case that $i=j$, i.e., the expert decision for that voxel was correct. Consequently, $\lambda_{i, i}^{(k)}$ is the usual sensitivity of expert $k$ for label $i$. We also note that for each expert $k$ the matrix $\left(\lambda_{i, j}^{(k)}\right)_{i, j}$ is a row-normalized version of the "confusion matrix" [1] in Bayesian multi-classifier algorithms. This matrix, when filled with proper coefficients, expresses prior knowledge about the decisions of each expert. In our algorithm, the coefficients are determined iteratively by an expectation maximization algorithm. 
In the "E" step of the EM algorithm, the unknown ground truth segmentation is estimated. Given the current estimate for the expert priors $(\lambda)$ and the expert decisions $e_{k}(x)$, the likelihood of voxel $x$ being in class $C_{i}$ is

$$
W\left(x \in C_{i}\right)=\frac{P\left(x \in C_{i}\right) \prod_{k} \lambda_{i, e_{k}(x)}^{(k)}}{\sum_{i^{\prime}}\left[P\left(x \in C_{i^{\prime}}\right) \prod_{k} \lambda_{i^{\prime}, e_{k}(x)}^{(k)}\right]} .
$$

Note that $W$ is a function of two parameters, $x$ and $i$. The "M" step of our algorithm estimates the expert priors $(\lambda)$ that maximize the likelihood of the current ground truth estimate determined in the preceding "E" step. Given the previous estimates $W$ of the class probabilities, the new estimates for the expert parameters are computed as follows:

$$
\hat{\lambda}_{i, j}^{(k)}=\frac{\sum_{x: e_{k}(x)=j} W\left(x \in C_{i}\right)}{\sum_{x} W\left(x \in C_{i}\right)} .
$$

\section{Evaluation Study Design}

The MNI BrainWeb Phantom 89] is used as the gold standard segmentation for the present study. More precisely, the phantom is used both with and without simulated multiple sclerosis lesions, resulting in two slightly different atlases. Each atlas consists of $220 \times 180 \times 220$ voxels with an isotropic resolution of $1 \mathrm{~mm}$. A slice from each of the two atlases is shown in Fig. 2. Individual segmentations by different experts are simulated by deforming the gold standard atlas with random deformations. Free-form deformations based on third-order B-splines [10, each with control point spacing of $40 \mathrm{~mm}$ and different Gaussian-distributed control point displacements, were applied to each atlas. The standard deviations of the Gaussian distributions were 1, 2, 3, and $4 \mathrm{~mm}$. For each standard deviation, thirty random deformation fields were generated. Each of the three classifier combination methods described above was used to compute combined segmentations from the same sets of $3,4,5,10,15,20,25$, and 30 randomly deformed atlases. Each result was compared to the original, undeformed atlas by counting the relative number of correctly labeled voxels.

\section{Results}

For each combined segmentation generated by the three methods under consideration (label averaging, binary EM, and multi-label EM) the average recognition rate of all labels, i.e., the percentage of correctly labeled voxels, was computed. The results are shown in Fig. 3, plotted over the number of experts included in the combined segmentation. For small numbers of experts (less than five), label averaging with no priors sometimes produced more accurate combined segmentations than either of the EM methods, but it did not benefit as much 


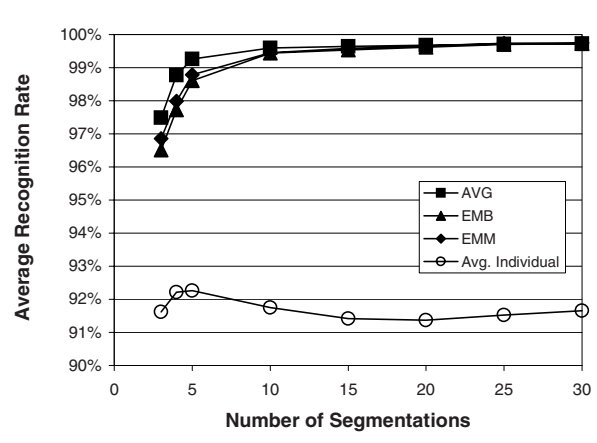

(a) $\sigma=1 \mathrm{~mm}$

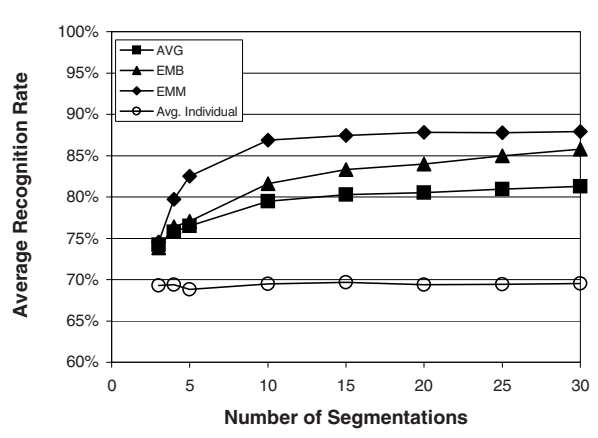

(c) $\sigma=3 \mathrm{~mm}$

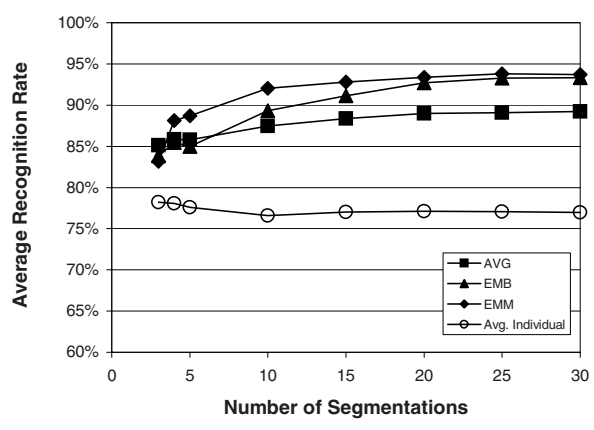

(b) $\sigma=2 \mathrm{~mm}$

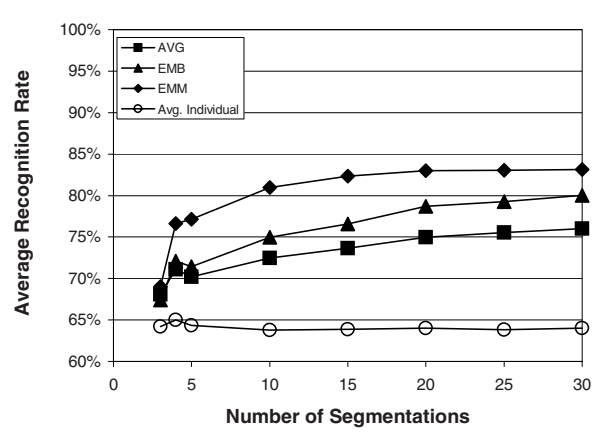

(d) $\sigma=4 \mathrm{~mm}$

Fig. 3. Segmentation accuracies (i.e., percentages of correctly classified voxels) over number of experts for three different decision fusion methods and four different random segmentation deformation magnitudes. The dashed line shows the average recognition rates of the respective set of individual experts, i.e., rate of correctly classified voxels by the individual experts, averaged over all experts.

from increasing the number of segmentations included. The results of both EM methods improved as more individual segmentations were added into the combination, and the multi-label EM method consistently outperformed the binary EM method. The differences between the three methods were larger, the bigger the magnitude of the random atlas deformations, i.e., the more different and inaccurate the individual segmentations.

Figure 3 also shows the average individual classification accuracy of the respective subset of experts included in the decision fusion. Since the experts were simulated by random deformations of the ground truth, the average individual classification accuracy is approximately constant and invariant under the number of expert. Two observations can easily be made when comparing the average individual accuracy to the accuracies achieved by decision fusion. First, the no- 


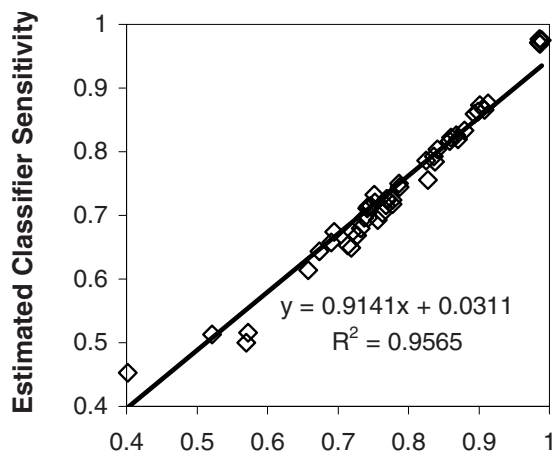

A-Posteriori Recognition Rate

(a) Binary EM

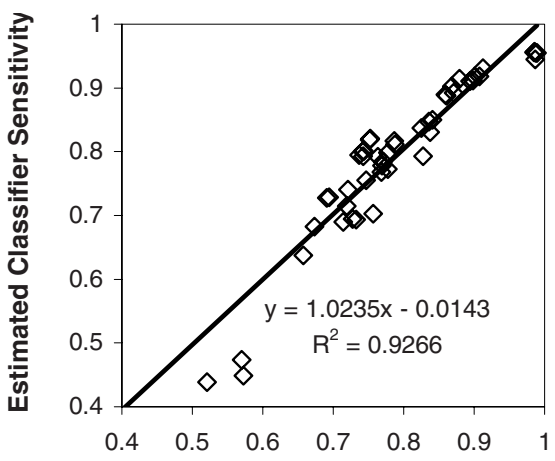

A-Posteriori Recognition Rate

(b) Multi-label EM

Fig. 4. Comparison of predicted and actual expert parameters. The Pearson correlation coefficients between estimated and true sensitivities are 0.978 for the binary EM algorithm and 0.963 for the multi-label EM algorithm.

priors label averaging method is always better than the individual experts. This is easily explained by the fact that the experts in this study are random and independent. Second, the methods using expert priors usually require a minimum number of experts in order to outperform the individual expert results. Beyond that threshold, both prior-based combination methods outperform the individual experts, in particular the multi-label EM algorithm with across-label priors.

As an illustration of the predictive accuracy of the EM methods, Fig. 4 compares the estimated classifier parameters (sensitivities) with the actual performances. Since the ground truth segmentation (the undeformed MNI atlas) is known in our study, we can compute the a posteriori recognition rates for all classes and all classifiers and compare these to the sensitivities estimated by the two EM methods. The plots, generated from five random atlases with deformation magnitude $\sigma=2 \mathrm{~mm}$, show excellent agreement between estimated and true classifier sensitivities for both EM methods.

\section{Discussion}

This paper has demonstrated that image segmentation can benefit from decision fusion, much like other classification problems. It has in particular presented evidence for the benefit of cross-label expert priors in the combination of multiple segmentations. The findings can be summarized as follows: there is valuable information in the misclassification statistics of segmentations of the human brain. These statistics can be exploited in the form of expert priors, which can, 
for example, be estimated by means of the multi-label EM algorithm introduced by our group [7.

As a final remark, and a word of caution, it should be noted that the classification accuracy of the combined expert decisions strongly depends on independent experts. Systematic errors that are shared among all (or most) of the experts will most likely propagate into the final classification outcome. The improvement of the individual segmentation accuracies of different labeling methods therefore remains an important goal.

Acknowledgments. TR was supported by the National Science Foundation under Grant No. EIA-0104114. DBR was supported by the Interdisciplinary Initiatives Program, which is part of the Bio-X Program at Stanford University, under the grant "Image-Guided Radiosurgery for the Spine and Lungs."

\section{References}

1. L. Xu, A. Krzyzak, C. Y. Suen, "Methods of combining multiple classifiers and their applications to handwriting recognition," IEEE Trans. Syst. Man Cybern., 22(3):418-435, 1992.

2. J. Kittler, M. Hatef, R. P. W. Duin, J. Matas, "On combining classifiers," IEEE Trans. Pattern Anal. Machine Intell., 20(3)226-239, 1998.

3. T. Rohlfing, R. Brandt, R. Menzel, C. R. Maurer, Jr., "Segmentation of threedimensional images using non-rigid registration: Methods and validation with application to confocal microscopy images of bee brains," in Medical Imaging: Image Processing, Feb. 2003, vol. 5032 of Proceedings of the SPIE, pp. 363-374.

4. B. M. Dawant, S. L. Hartmann, J. P. Thirion, F. Maes, D. Vandermeulen, P. Demaerel, "Automatic 3-D segmentation of internal structures of the head in MR images using a combination of similarity and free-form transformations: Part I, methodology and validation on normal subjects," IEEE Trans. Med. Imag., 18(10):909-916, 1999.

5. S. K. Warfield, K. H. Zou, W. M. Wells, "Validation of image segmentation and expert quality with an expectation-maximization algorithm," in Proceedings of Fifth International Conference on Medical Image Computing and Computer-Assisted Intervention (MICCAI), Part I, vol. 2488 of LNCS, pp. 298-306, Springer-Verlag, Berlin, 2002.

6. A. Verikas, A. Lipnickas, K. Malmqvist, M. Bacauskiene, A. Gelzinis, "Soft combination of neural classifiers: A comparative study," Pattern Recogn. Lett., 20(4):429444, 1999.

7. T. Rohlfing, D. B. Russakoff, C. R. Maurer, Jr., "Expectation maximization strategies for multi-atlas multi-label segmentation," in Information Processing in Medical Imaging, vol. 2732 of LNCS, pp. 210-221, Springer-Verlag, Berlin, 2003.

8. Online: http://www.bic.mni.mcgill.ca/brainweb/

9. D. L. Collins, A. P. Zijdenbos, V. Kollokian, J. G. Sled, N. J. Kabani, C. J. Holmes, A. C. Evans, "Design and construction of a realistic digital brain phantom," IEEE Trans. Med. Imag., 17(3):463-468, 1998.

10. T. W. Sederberg, S. R. Parry, "Free-form deformation and solid geometric models," Comput. Graph. (ACM), 20(4):151-160, 1986. 